\title{
The Kuramoto model in presence of additional interactions that break rotational symmetry
}

\author{
V K Chandrasekar ${ }^{1}$, M Manoranjani ${ }^{1}$ and Shamik Gupta ${ }^{2,3}$ \\ ${ }^{1}$ Centre for Nonlinear Science 85 Engineering, School of Electrical \& Electronics Engineering, \\ SASTRA Deemed University, Thanjavur-613 401, Tamil Nadu, India \\ ${ }^{2}$ Department of Physics, Ramakrishna Mission Vivekananda \\ Educational and Research Institute, Belur Math, Howrah 711202, India \\ ${ }^{3}$ Regular Associate, Quantitative Life Sciences Section, \\ ICTP - The Abdus Salam International Centre for Theoretical Physics, Strada Costiera 11, 34151 Trieste, Italy
}

(Dated: July 14, 2020)

\begin{abstract}
The Kuramoto model serves as a paradigm to study the phenomenon of spontaneous collective synchronization. We study here a nontrivial generalization of the Kuramoto model by including an interaction that breaks explicitly the rotational symmetry of the model. In an inertial frame (e.g., the laboratory frame), the Kuramoto model does not allow for a stationary state, that is, a state with time-independent value of the so-called Kuramoto (complex) synchronization order parameter $z \equiv r e^{i \psi}$; Note that a time-independent $z$ implies $r$ and $\psi$ both time independent, with the latter fact corresponding to a state in which $\psi$ rotates at zero frequency (no rotation). In this backdrop, we ask: Does the introduction of the symmetry-breaking term suffice to allow for the existence of a stationary state in the laboratory frame? Compared to the original model, we reveal a rather rich phase diagram of the resulting model, with the existence of both stationary and standing wave phases. While in the former the synchronization order parameter $r$ has a long-time value that is time independent, one has in the latter an oscillatory behavior of the order parameter as a function of time that nevertheless yields a non-zero and time-independent time average. Our results are based on numerical integration of the dynamical equations as well as an exact analysis of the dynamics by invoking the so-called Ott-Antonsen ansatz that allows to derive a reduced set of time-evolution equations for the order parameter.
\end{abstract}

Keywords: Spontaneous synchronization, Kuramoto model, Bifurcation

\section{INTRODUCTION: MODEL AND SUMMARY OF RESULTS}

One of the widely invoked and extensively studied models of spontaneous collective synchronization in the field of nonlinear dynamics is the so-called Kuramoto model [1]. The setting of the model allows to apply it to study a wide range of physical systems pervading length and time scales of several order of magnitude, ranging from groups of fireflies flashing on and off in unison [2], cardiac pacemaker cells [3], electrochemical [4 and electronic [5] oscillators, to Josephson junction arrays [6], audience clapping in unison [7, electrical power-grid networks [8], in discussing adaptive networks in neuroscience and social sciences [9], etc. The model comprises nearlyidentical $N$ limit-cycle oscillators with distributed natural frequencies $\omega_{j} ; j=1,2, \ldots, N$, interacting weakly with one another, with the strength of coupling being the same for every pair of oscillators [10 14]. Denoting by $\theta_{j} \in[-\pi, \pi]$ the phase of the $j$-th oscillator, the dynamics of the model is described by a set of $N$ coupled first-order nonlinear differential equations of the form

$$
\frac{\mathrm{d} \theta_{j}}{\mathrm{~d} t}=\omega_{j}+\frac{K}{N} \sum_{k=1}^{N} \sin \left(\theta_{k}-\theta_{j}\right),
$$

where $K \geq 0$ is the coupling constant. The frequencies $\left\{\omega_{j}\right\}_{1 \leq j \leq N}$ denote a set of quenched-disordered random variables distributed according to a common distribution $g(\omega)$, with normalization $\int_{-\infty}^{\infty} \mathrm{d} \omega g(\omega)=1$ and finite mean $\omega_{0}$. The Kuramoto synchronization order parameter, giving a measure of synchrony present in the system at time $t$, is defined as 11

$$
\begin{aligned}
& z(t)=r(t) e^{\mathrm{i} \psi(t)} \equiv \frac{1}{N} \sum_{j=1}^{N} e^{\mathrm{i} \theta_{j}(t)}=r_{x}(t)+\mathrm{i} r_{y}(t) \\
& \left(r_{x}, r_{y}\right)(t) \equiv \frac{1}{N} \sum_{j=1}^{N}\left(\cos \theta_{j}, \sin \theta_{j}\right)(t) .
\end{aligned}
$$

The quantity $r(t) ; 0 \leq r(t) \leq 1$, measures the amount of synchrony present in the system at time $t$, while $\psi(t)=\tan ^{-1}\left(r_{y}(t) / r_{x}(t)\right)$ gives the average phase. When the oscillators are incoherent or unsynchronized so that over a stretch of time or in an ensemble of $\theta_{j}$-values at a given time, one has with equal probabilities $e^{\mathrm{i} \theta_{j}}$ for any $j$ equal to any complex number with modulus unity, $r(t)$ averages to zero. On the other hand, $r(t)$ has a non-zero average in the synchronized phase in which a finite fraction of oscillators have phase differences that are constant in time.

The dynamics (1) satisfies rotational symmetry, whereby rotating every phase by an arbitrary angle same for all leaves the dynamics invariant. In particular, one may implement the transformation $\theta_{j}(t) \rightarrow$ $\theta_{j}(t)+\omega_{0} t \forall j, t$, which is tantamount to viewing the dynamics in a frame rotating uniformly with frequency 
$\omega_{0}$ with respect to an inertial frame, e.g., the laboratory frame. In such a comoving frame, the frequencies $\omega_{j}$ follow the shifted distribution $g\left(\omega+\omega_{0}\right)$, thereby having zero mean.

The model (1) has been extensively studied over the years and a host of results have been derived, see Ref. [14] for a recent overview. For example, consider a $g(\omega)$ that is unimodal, namely, a $g(\omega)$ that is symmetric about its mean $\omega_{0}$ and decreases monotonically and continuously to zero with increasing $\left|\omega-\omega_{0}\right|$. Considering the limit $N \rightarrow \infty$, it is then known that in the stationary state of the dynamics (1), attained in the limit $t \rightarrow \infty$ and in the comoving frame, the system may exist in either a synchronized or an incoherent phase depending on whether the coupling $K$ is respectively above or below a critical threshold $K_{c}=2 /\left(\pi g\left(\omega+\omega_{0}\right)\right)$. On tuning $K$ across $K_{c}$ from high to low values, one observes a continuous phase transition in $r_{\mathrm{st}}$, the stationary value of $r(t)$; Namely, $r_{\mathrm{st}}$ decreases continuously from the value of unity, achieved as $K \rightarrow \infty$, to zero at $K=K_{c}$ and remains zero at smaller $K$ values. It is then usual to interpret the transition as the case of a supercritical bifurcation, in which on tuning $K$ from low to high values, a synchronized phase bifurcates from the incoherent phase at $K=K_{c}$. In particular, a small change of $K$ across $K_{c}$ results in only a small change in the value of $r_{\mathrm{st}}$. The transition could also be of first order (e.g., for a bimodal $g(\omega)$ [15] or in the inertial version of the dynamics (1) [13), whereby $r_{\mathrm{st}}$ exhibits an abrupt and big change on changing $K$ by a small amount across the phase transition point; in this case, the bifurcation is said to be subcritical and leads to hysteresis [14. For discussions on general form of the Kuramoto model and arbitrary frequency distributions, see, e.g., Refs. [16, 17.

In this work, we consider a generalization of the $\mathrm{Ku}$ ramoto dynamics (1) by including an interaction term that explicitly breaks the rotational symmetry of the dynamics. To this end, we consider the following set of $N$ coupled nonlinear differential equations:

$\frac{\mathrm{d} \theta_{j}}{\mathrm{~d} t}=\omega_{j}+\frac{1}{N}\left[\epsilon_{1} \sum_{k=1}^{N} \sin \left(\theta_{k}-\theta_{j}\right)+\epsilon_{2} \sum_{k=1}^{N} \sin \left(\theta_{k}+\theta_{j}\right)\right]$,

where the real parameters $\epsilon_{1,2}$ denote the coupling constants. In terms of the quantities $r_{x}, r_{y}$, Eq. (3), the dynamics (4) reads

$$
\frac{\mathrm{d} \theta_{j}}{\mathrm{~d} t}=\omega_{j}+\left(\epsilon_{1}+\epsilon_{2}\right) r_{y} \cos \theta_{j}+\left(\epsilon_{2}-\epsilon_{1}\right) r_{x} \sin \theta_{j} .
$$

The above equation makes it evident that the quantities $r_{x}$ and $r_{y}$ act as mean-fields determining the motion of every oscillator in the ensemble. In Appendix $\mathrm{A}$ we motivate the form of the dynamics (4) by considering the dynamics of a collection of $N$ globally-coupled StuartLandau limit-cycle oscillators with conjugate feedback.

Note that setting $\epsilon_{2}=0$ in Eq. (4) (equivalently, Eq. (5p) reduces the dynamics to that of the Kuramoto model (1) on identifying $\epsilon_{1}$ with the parameter $K \geq 0$.
We therefore take $\epsilon_{1}$ to be positive. Then, for a fixed $\epsilon_{1}$, changing $\epsilon_{2}$ to $-\epsilon_{2}$ is tantamount to keeping $\epsilon_{2}$ unchanged but effecting the transformation $\theta_{j} \rightarrow \theta_{j}+$ $\pi / 2 \forall j$ in Eq. (4) (redefinition of the origin with respect to which the phases are measured). Consequently, we may take $\epsilon_{2} \geq 0$, without loss of generality. Rotational symmetry is possible in the dynamics (4) only with the choice $\epsilon_{2}=0$, so that the $\epsilon_{2}$-term in the dynamics may be interpreted as a rotational-symmetry-breaking interaction. In contrast to the Kuramoto model, the dynamics (4) is not invariant with respect to the transformation $\theta_{j} \rightarrow \theta_{j}^{\prime} \equiv \theta_{j}+\omega_{0} t \forall j, t$ because of the $\epsilon_{2}$-term. Indeed, under such a transformation, we get

$$
\begin{aligned}
\frac{\mathrm{d} \theta_{j}^{\prime}}{\mathrm{d} t}= & \omega_{j}+\omega_{0}+\frac{1}{N}\left[\epsilon_{1} \sum_{k=1}^{N} \sin \left(\theta_{k}^{\prime}-\theta_{j}^{\prime}\right)\right. \\
& \left.+\epsilon_{2} \sum_{k=1}^{N} \sin \left(\theta_{k}^{\prime}+\theta_{j}^{\prime}-2 \omega_{0} t\right)\right],
\end{aligned}
$$

which does not have the same form as the dynamics (4), and so the transformation does not leave the dynamics invariant. As a result, the mean $\omega_{0}$ is expected to have an essential effect on the dynamics (4), which cannot be gotten rid of by viewing the dynamics in a frame rotating uniformly with frequency $\omega_{0}$ with respect to the laboratory frame, as is possible with the Kuramoto model. From now on, we will study the dynamics (4) only in the inertial frame (i.e., the laboratory frame) and not in the comoving frame.

As is usual with studies of the Kuramoto model, we will consider in this work a unimodal $g(\omega)$. Specifically, we will consider two representative choices, namely, a Lorentzian:

$$
g(\omega)=\frac{\gamma}{\pi\left(\left(\omega-\omega_{0}\right)^{2}+\gamma^{2}\right)} ; \gamma>0
$$

and a Gaussian:

$$
g(\omega)=\frac{1}{\sqrt{2 \pi \sigma^{2}}} \exp \left(-\left(\omega-\omega_{0}\right)^{2} /\left(2 \sigma^{2}\right)\right) ; \quad \sigma^{2}>0 .
$$

Let us remark on a relevant aspect of the dynamics (4). Summing both sides of the equation over $j$, we get in the limit $N \rightarrow \infty$ that the mean ensemble frequency of the $\theta_{j}$ 's is given by

$$
f \equiv \frac{\mathrm{d}}{\mathrm{d} t}\left(\frac{1}{N} \sum_{j=1}^{N} \theta_{j}\right)=\omega_{0}+2 \epsilon_{2} r_{x} r_{y}
$$

where we have used the fact that $\lim _{N \rightarrow \infty}(1 / N) \sum_{j=1}^{N} \omega_{j}=\omega_{0}$. From Eq. 99, we see that the mean ensemble frequency $f$ coincides with the mean of the natural frequency distribution $g(\omega)$ when the dynamics (4) becomes that of the Kuramoto model (that is, $\epsilon_{2}=0$ ). In our case, with $\epsilon_{2} \neq 0$, the two frequencies would in general not coincide unless the system is unsynchronized so that $r_{x}=r_{y}=0$. 
Considering the limit $N \rightarrow \infty$, this work aims at a detailed characterization of the long-time $(t \rightarrow \infty)$ limit of the dynamics (4), equivalently, Eq. (5), with the primary objective of identifying and understanding what new features are brought in by the introduction of the rotational-symmetry-breaking $\epsilon_{2}$-term. Now, in the absence of the $\epsilon_{2}$-term, the dynamics does not allow for a stationary state in the laboratory frame. Namely, one cannot have in such a frame time-independent $z$ : in the synchronized phase, $\psi$ will change uniformly in time with frequency $\omega_{0}$. Note that a time-independent $z$ implies $r$ and $\psi$ both time independent, with the latter fact corresponding to a state in which $\psi$ rotates at zero frequency (no rotation). In this backdrop, we ask: Does the introduction of the $\epsilon_{2}$-term suffice to allow for the existence of a stationary state in the laboratory frame? If the answer is in the affirmative, some immediate and pertinent questions follow: What is the nature of the stationary state? Is there a range of parameter values for which one has a synchronized stationary state? What is the complete phase diagram in the $\left(\epsilon_{1}-\epsilon_{2}\right)$-plane? Can one characterize the phase diagram analytically?

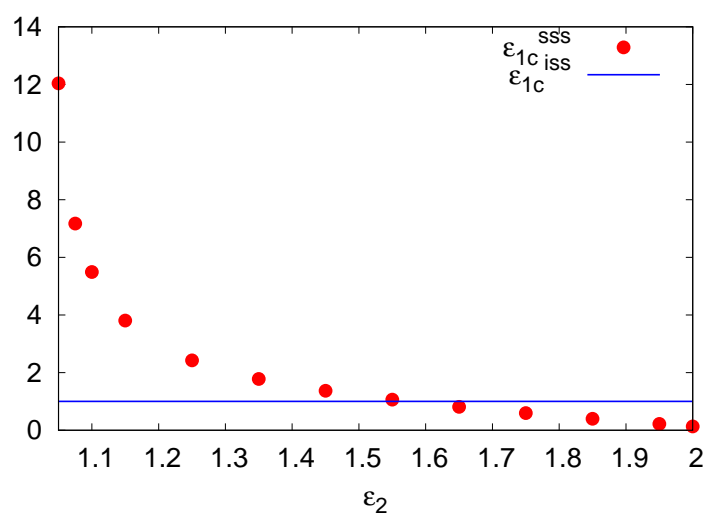

FIG. 1. For the model (4) and considering for $\omega_{j}$ 's the Lorentzian distribution (7) with $\gamma=0.5$ and $\omega_{0}=1.0$, the figure shows the thresholds $\epsilon_{1 c}^{\mathrm{ISS}}\left(\epsilon_{2}\right)$ and $\epsilon_{1 c}^{\mathrm{SSS}}\left(\epsilon_{2}\right)$ as a function of $\epsilon_{2}$, where $\epsilon_{1 c}^{\mathrm{ISS}}\left(\epsilon_{2}\right)$ (respectively, $\epsilon_{1 c}^{\mathrm{SSS}}\left(\epsilon_{2}\right)$ ) defines the stability threshold of an incoherent stationary state (ISS) (respectively, the existence threshold of a synchronized stationary state $(\mathrm{SSS}))$.

We will unravel in this paper a rather rich phase diagram exhibited by the dynamics (4). We will show that for a given $\epsilon_{2} \neq 0$, a stationary state (namely, a state for which $z$ is stationary) occurs only for $\epsilon_{1}<\epsilon_{1 c}^{\text {ISS }}\left(\epsilon_{2}\right)$ and for $\epsilon_{1}>\epsilon_{1 c}^{\mathrm{SSS}}\left(\epsilon_{2}\right)$, where $\epsilon_{1 c}^{\mathrm{ISS}}\left(\epsilon_{2}\right)$ (respectively, $\epsilon_{1 c}^{\mathrm{SSS}}\left(\epsilon_{2}\right)$ ) defines the stability threshold of an incoherent stationary state (ISS) (respectively, the existence threshold of a synchronized stationary state (SSS)). The relative magnitude of $\epsilon_{1 c}^{\mathrm{ISS}}\left(\epsilon_{2}\right)$ and $\epsilon_{1 c}^{\mathrm{SSS}}\left(\epsilon_{2}\right)$ depends on the value of $\epsilon_{2}$, the relative ordering is reversed. Figure 1 shows, based on our analysis presented later in the paper, representative results for $\epsilon_{1 c}^{\mathrm{ISS}}\left(\epsilon_{2}\right)$ and $\epsilon_{1 c}^{\mathrm{SSS}}\left(\epsilon_{2}\right)$ for a Lorentzian $g(\omega)$, Eq. (7). In Fig. 2, we show in the $\left(\epsilon_{1}-\epsilon_{2}\right)$-plane
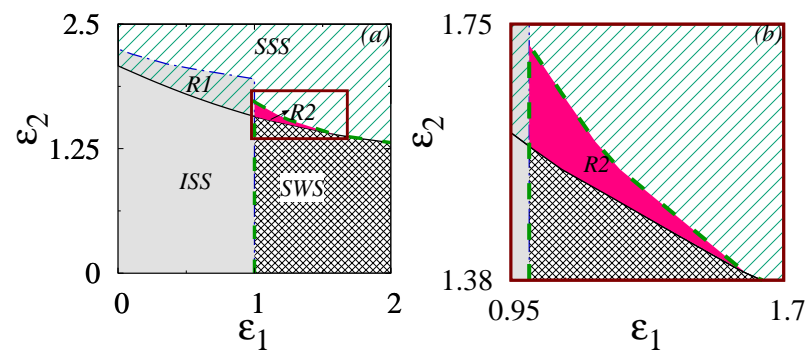

FIG. 2. For the model (4) and considering for $\omega_{j}$ 's the Lorentzian distribution (7) with $\gamma=0.5$ and $\omega_{0}=1.0$, the figure depicts the two-parameter bifurcation diagram in the $\left(\epsilon_{1}, \epsilon_{2}\right)$-plane. We show here the various stable phases and the phase boundaries, with bifurcation behavior observed as one crosses the phase boundaries. (a) The shaded regions, representing stable existence of the ISS (Incoherent Stationary State), the SSS (Synchronized Stationary State) and the SWS (Standing Wave State), have been constructed by analyzing the long-time numerical solution of the dynamics (4) for $N=10^{5}$. In numerics, we distinguish between the different regions by requiring that at long times, $r(t)$ as a function of $t$ behaves differently for the ISS, the SSS and the SWS. Namely, for the ISS, both the order parameter $r(t)$ and its time average take the value zero in the $t \rightarrow \infty$ limit. In the SWS, $r(t)$ as $t \rightarrow \infty$ oscillates in time around a timeindependent non-zero value, thereby yielding a non-zero time average. For the SSS, too, $r(t)$ as $t \rightarrow \infty$ has a non-zero timeindependent time average, but it does not oscillate, instead remains equal to a nonzero constant in time. The regions R1 and R2 represent multistability (hysteresis) between ISS-SSS and SSS-SWS, respectively. The dot-dashed blue line is obtained by using Eq. (27), the solid black line is obtained by using Eq. 29, while the dashed green line is obtained from an analysis of Eq. (23) by using the numerical package XPPAUT [18] in which we study the stability of the SWS; Note that all these lines are based on the Ott-Ansatz-reduced dynamics corresponding to the dynamics (4). (b) Enlarged view of the boxed region in (a).

the various stable phases for the model (4) and the phase boundaries. The different phases are distinguished on the basis of the long-time behavior of the order parameter $r(t)$. Namely, for the ISS, one has in the long-time $(t \rightarrow \infty)$ limit both the order parameter $r(t)$ and its time average, defined as

$$
R \equiv \lim _{t \rightarrow \infty} \frac{1}{\tau} \int_{t}^{t+\tau} \mathrm{d} t^{\prime} r\left(t^{\prime}\right),
$$

taking the value zero; thus, we have $r_{\text {st }}=0$, and also, $R=0$. Note that for the ISS, we have $\mathrm{d} \psi / \mathrm{d} t \neq 0$ at long times. In the standing wave state (SWS), $r(t)$ as $t \rightarrow \infty$ oscillates in time around a time-independent non-zero value. Thus, in this case, $r(t)$ does not have a stationary value, but we have $R \neq 0$; also, $\mathrm{d} \psi / \mathrm{d} t \neq 0$ at long times. For the SSS, too, $R \neq 0$, but $r(t)$ here does not oscillate, instead remains equal to a non-zero constant in time. For the SSS, $\psi$ at long times assumes a time-independent value so that $\mathrm{d} \psi / \mathrm{d} t=0$ at long times. On the basis of the 
foregoing, we summarize that the mean-field frequency $\Omega \equiv \mathrm{d} \psi / \mathrm{d} t$ is zero for the SSS, while the same for the ISS and the SWS have time-dependent values. The regions $\mathrm{R} 1$ and R2 in the phase diagram 2 represent multistability (hysteresis) between ISS-SSS and SSS-SWS, respectively. At a fixed $\epsilon_{1}$ and on tuning $\epsilon_{2}$ (or vice versa), one observes phase transitions/bifurcations as one crosses the different phase boundaries. Figure 2, the phase diagram or the bifurcation diagram of model (4), is the key result of our work. To summarize the nature of the different displayed phases, we have:

- ISS: $r_{\mathrm{st}}=0, R=0$.

- SWS: $r(t)$ oscillates with time, $r_{\mathrm{st}}$ does not exist, $R \neq 0$.

- SSS: $r_{\mathrm{st}} \neq 0, R \neq 0$.

Thus, only the ISS and the SSS qualify as a stationary state (time-independent $z$ ). With respect to Fig. 2, we note that the phase diagram contains regions of both first-order and continuous transitions. As usual, the former is the case whenever on tuning the parameters, one encounters a region of multistability in going from one phase to another in the phase diagram, while a continuous transition happens in the absence of encountering a region of multistability. For $\epsilon_{2}=0$, when our model (4) reduces to the Kuramoto model, one has however only a continuous and no first-order transition.

The rest of the paper is devoted to a derivation of the aforementioned results. For Lorentzian $g(\omega)$, Eq. (7), we use exact analytical results derived by applying the so-called Ott-Antonsen (OA) ansatz, combined with numerical integration of the dynamics (4) for large $N$, to support the bifurcation diagram of Fig. 2. For the Gaussian $g(\omega)$, Eq. 8), we present numerical results to suggest existence of similar stable phases as in the case of the Lorentzian. The OA ansatz allows to rewrite in the limit $N \rightarrow \infty$ the dynamics of coupled networks of phase oscillators in terms of a few collective variables [19, 20]. The power of the ansatz, which explains its usefulness and its wide applicability, lies in its remarkable ability to capture precisely and quantitatively, through the dynamical equations for these collective variables, all, and not just some, of the order parameter attractors and bifurcations of the dynamics. The latter features may be obtained directly by performing numerical integration of the defining equations of motion for $N \gg 1$ and evaluating $r(t)$ in numerics.

The paper is organized as follows. In the next section, we discuss the OA-ansatz-reduced dynamical equations for the model (4) for the choice of the Lorentzian $g(\omega)$, Eq. (7), while in Section III, we discuss the implications of the reduced dynamics for the existence of the incoherent and the synchronized stationary state as well as the standing wave state. In Section IV] we present and discuss results obtained from numerical integration of the dynamics (4) for the Lorentzian and the Gaussian $g(\omega)$,
Eqs. (7) and (8), respectively, and compare for the former the numerical and the OA-ansatz-based analytical results. The paper ends with conclusions in Section V.

\section{ANALYSIS OF THE DYNAMICS (4): THE OTT-ANTONSEN (OA) ANSATZ}

We now provide an analysis of the dynamics (4), equivalently, the dynamics (5), in the limit $N \rightarrow \infty$, by invoking the OA ansatz. In this limit, the dynamics (5) may be characterized by the single-oscillator distribution function $f(\theta, \omega, t)$, defined such that $f(\theta, \omega, t) \mathrm{d} \theta$ gives the probability out of oscillators with natural frequency $\omega$ to find an oscillator with phase in the range $[\theta, \theta+\mathrm{d} \theta]$ at time $t$. The distribution is $2 \pi$-periodic in $\theta$ and obeys the normalization

$$
\int_{0}^{2 \pi} \mathrm{d} \theta f(\theta, \omega, t)=g(\omega) \forall \omega
$$

The $N \rightarrow \infty$ generalization of Eq. (3) reads

$$
\left(r_{x}, r_{y}\right)(t) \equiv \int \mathrm{d} \omega \int_{0}^{2 \pi} \mathrm{d} \theta(\cos \theta, \sin \theta) f(\theta, \omega, t) g(\omega) .
$$

Since the dynamics (5) conserves the number of all oscillators with a given $\omega$, the time evolution of $f$ follows the continuity equation

$\frac{\partial f}{\partial t}+\frac{\partial}{\partial \theta}\left[\left(\omega+\left(\epsilon_{1}+\epsilon_{2}\right) r_{y} \cos \theta+\left(\epsilon_{2}-\epsilon_{1}\right) r_{x} \sin \theta\right) f\right]=0$

Being $2 \pi$-periodic in $\theta$, one may effect a Fourier expansion of $f$ as

$$
f(\theta, \omega, t)=\frac{g(\omega)}{2 \pi}\left[1+\sum_{n=1}^{\infty}\left(\alpha_{n}(\omega, t) e^{\mathrm{i} n \theta}+\text { c.c. }\right)\right]
$$

where the prefactor of $g(\omega)$ ensures that the normalization (11) is satisfied, $\alpha_{n}(\omega, t)$ is the $n$-th Fourier coefficient, while c.c. denotes the term obtained by complex conjugation of the first term within the brackets. The OA ansatz consists in assuming [19, 20]

$$
\alpha_{n}(\omega, t)=[\alpha(\omega, t)]^{n},
$$

where the arbitrary function $\alpha(\omega, t)$ is assumed to satisfy $|\alpha(\omega, t)|<1$, together with the requirements that $\alpha(\omega, t)$ may be analytically continued to the whole of the complex- $\omega$ plane, it has no singularities in the lower-half complex- $\omega$ plane, and $|\alpha(\omega, t)| \rightarrow 0$ as $\operatorname{Im}(\omega) \rightarrow-\infty$.

Using the choice (15) in Eq. (14) defines in the space $\mathcal{D}$ of all possible distributions $f(\theta, \omega, t)$ a particular class defined on a manifold $\mathcal{M}$ in $\mathcal{D}$. Originally implemented in the context of the Kuramoto model for a Lorentzian distribution of the oscillator frequencies, it was shown that this particular class of $f$ remains confined to the manifold $\mathcal{M}$ under the time evolution and yields a single 
first-order ordinary differential equation for the evolution of the synchronization order parameter $r(t)$ [19, 20].
In order to proceed with the OA-ansatz-analysis of the dynamics (5), we consider a Lorentzian $g(\omega)$, see Eq. (7). Using Eqs. (14) and (15) in Eq. (12) yields

$$
\begin{aligned}
& r_{x}=\frac{1}{2} \int_{-\infty}^{\infty} \mathrm{d} \omega g(\omega)\left(\alpha^{\star}(\omega, t)+\alpha(\omega, t)\right)=\frac{1}{4 \mathrm{i} \pi} \oint_{C} \mathrm{~d} \omega\left[\frac{1}{\left(\omega-\omega_{0}\right)-\mathrm{i} \gamma}-\frac{1}{\left(\omega-\omega_{0}\right)+\mathrm{i} \gamma}\right]\left[\alpha^{\star}(\omega, t)+\alpha(\omega, t)\right], \\
& r_{y}=\frac{1}{2 \mathrm{i}} \int_{-\infty}^{\infty} \mathrm{d} \omega g(\omega)\left(\alpha^{\star}(\omega, t)-\alpha(\omega, t)\right)=-\frac{1}{4 \pi} \oint_{C} \mathrm{~d} \omega\left[\frac{1}{\left(\omega-\omega_{0}\right)-\mathrm{i} \gamma}-\frac{1}{\left(\omega-\omega_{0}\right)+\mathrm{i} \gamma}\right]\left[\alpha^{\star}(\omega, t)-\alpha(\omega, t)\right],
\end{aligned}
$$

where $\star$ denotes complex conjugation, and where the contour $C$ consists of the $\operatorname{Re}(\omega)$-axis closed by a large semicircle in the lower-half complex- $\omega$ plane. In obtaining the second equality in Eqs. (16) and (17), we have used the form (7) and the fact that the contribution to the contour integral from the semicircular part of the contour vanishes in view of $|\alpha(\omega, t)| \rightarrow 0$ as $\operatorname{Im}(\omega) \rightarrow-\infty$. Evaluating the above integrals by the residue theorem, we get

$$
\begin{aligned}
& r_{x}=\frac{\alpha\left(\left(\omega_{0}-\mathrm{i} \gamma\right), t\right)+\alpha^{\star}\left(\left(\omega_{0}-\mathrm{i} \gamma\right), t\right)}{2}, \\
& r_{y}=\frac{\alpha^{\star}\left(\left(\omega_{0}-\mathrm{i} \gamma\right), t\right)-\alpha\left(\left(\omega_{0}-\mathrm{i} \gamma\right), t\right)}{2 \mathrm{i}} .
\end{aligned}
$$

On the other hand, using the expansion (14) and the ansatz (15) in Eq. (13) and collecting and equating the coefficient of $e^{i \theta}$ to zero give

$$
\begin{aligned}
& \frac{\partial \alpha(\omega, t)}{\partial t}+\mathrm{i} \omega \alpha(\omega, t)+\frac{\epsilon_{1}+\epsilon_{2}}{2} \mathrm{i} r_{y}\left[1+\alpha^{2}(\omega, t)\right] \\
& +\frac{\epsilon_{2}-\epsilon_{1}}{2} r_{x}\left[1-\alpha^{2}(\omega, t)\right]=0 .
\end{aligned}
$$

Using Eqs. (3) and (18), the Kuramoto order parameter is obtained as

$$
z=\alpha^{\star}\left(\left(\omega_{0}-\mathrm{i} \gamma\right), t\right)
$$

Equation (19) then gives

$$
\frac{\partial z}{\partial t}-\mathrm{i}\left(\omega_{0}+\mathrm{i} \gamma\right) z-\frac{\epsilon_{1}+\epsilon_{2}}{2} \mathrm{i} r_{y}\left[1+z^{2}\right]+\frac{\epsilon_{2}-\epsilon_{1}}{2} r_{x}\left[1-z^{2}\right]=0,
$$

which on using Eq. 18 gives

$$
\frac{\partial z}{\partial t}-\mathrm{i}\left(\omega_{0}+\mathrm{i} \gamma\right) z-\frac{\epsilon_{1}}{2}\left(z-|z|^{2} z\right)+\frac{\epsilon_{2}}{2}\left(z^{\star}-z^{3}\right)=0
$$

Equation 22 rewritten in terms of the quantities $r$ and $\psi$, see Eq. (2), gives the following two coupled equations:

$$
\begin{aligned}
& \frac{\mathrm{d} r}{\mathrm{~d} t}=-\gamma r+r\left(1-r^{2}\right)\left(\frac{\epsilon_{1}}{2}-\frac{\epsilon_{2}}{2} \cos (2 \psi)\right), \\
& \frac{\mathrm{d} \psi}{\mathrm{d} t}=\omega_{0}+\frac{\epsilon_{2}}{2}\left(1+r^{2}\right) \sin (2 \psi) .
\end{aligned}
$$

The above equations constitute the OA-ansatz-reduced order parameter dynamics corresponding to the dynamics (5) in the limit $N \rightarrow \infty$. Note that for $\epsilon_{2}=0$, when one has the Kuramoto model, the two equations in (23) are decoupled, and there is only uniform rotation of $\psi$ with frequency $\omega_{0}$, that is, the mean-field frequency equals $\omega_{0}$; this case was analyzed in Ref. [19]. For $\epsilon_{2} \neq 0$, however, the situation is much more intricate, as we show below.

\section{ANALYSIS OF THE OA-ANSATZ-REDUCED DYNAMICS}

\section{A. Incoherent stationary state (ISS):}

The dynamics 22, equivalently the dynamics 23, allows for an incoherent stationary state (ISS) given by $z=z^{\star}=0$ for all values of $\epsilon_{1}$ and $\epsilon_{2}$; correspondingly, one has $r_{\mathrm{st}}=0$, and hence, $R=0$. The linear stability of this state is determined by linearizing Eq. (22) around $z=0$, by using the expansion $z=u$ with $|u| \ll 1$. To this end, we obtain the linear equation

$$
\frac{\partial u}{\partial t}-\mathrm{i}\left(\omega_{0}+\mathrm{i} \gamma-\mathrm{i} \frac{\epsilon_{1}}{2}\right) u+\frac{\epsilon_{2}}{2} u^{\star}=0
$$

Writing $u=u_{x}+\mathrm{i} u_{y}$ yields

$\frac{\partial}{\partial t}\left[\begin{array}{l}u_{x} \\ u_{y}\end{array}\right]=M\left[\begin{array}{l}u_{x} \\ u_{y}\end{array}\right] ; M \equiv\left[\begin{array}{cc}-\gamma+\frac{\epsilon_{1}}{2}-\frac{\epsilon_{2}}{2} & -\omega_{0} \\ \omega_{0} & -\gamma+\frac{\epsilon_{1}}{2}+\frac{\epsilon_{2}}{2}\end{array}\right]$

The matrix $M$ has eigenvalues

$$
\lambda_{1,2}=\frac{-2 \gamma+\epsilon_{1} \pm \sqrt{\Delta}}{2}
$$

with $\Delta \equiv \epsilon_{2}^{2}-4 \omega_{0}^{2}$. For a given $\epsilon_{2}$, the stability threshold for the ISS is then obtained as

$$
\begin{aligned}
& \text { (i) } \epsilon_{1 c}^{\mathrm{ISS}}\left(\epsilon_{2}\right)=2 \gamma \text { for } \Delta<0 \\
& \text { (ii) } \epsilon_{1 c}^{\mathrm{ISS}}\left(\epsilon_{2}\right)=2 \gamma-\sqrt{\Delta} \text { for } \Delta>0 .
\end{aligned}
$$




\section{B. Synchronized stationary state (SSS):}

Considering the dynamics 23, we now explore the possibility of existence of a synchronized stationary state (SSS), i.e., $r_{\mathrm{st}} \neq 0$, and hence, $R \neq 0$. In the case of the Kuramoto model, this would mean to have in the laboratory frame a state with time-independent $r$ together with $\psi$ changing uniformly in time with frequency $\omega_{0}$; in this case, on transforming to a frame rotating uniformly with frequency $\omega_{0}$ with respect to the laboratory frame, one obtains the SSS in which both $\psi$ and $r$ and, hence, $z$ have time-independent values. Considering the dynamics (23) and requiring $r$ and $\psi$ to have time-independent non-zero values $\left(r_{\mathrm{st}}, \psi_{\mathrm{st}}\right)$ so that the left hand side of the two equations in (23) may be set to zero, we obtain for the SSS the two coupled equations

$$
\begin{aligned}
& \frac{\epsilon_{2}}{2} \cos \left(2 \psi_{\mathrm{st}}\right)=\frac{\gamma}{\left(r_{\mathrm{st}}^{2}-1\right)}+\frac{\epsilon_{1}}{2}, \\
& \frac{\epsilon_{2}}{2} \sin \left(2 \psi_{\mathrm{st}}\right)=\frac{-\omega_{0}}{\left(1+r_{\mathrm{st}}^{2}\right)} .
\end{aligned}
$$

The above equations yield the following solutions for $\left(r_{\mathrm{st}}, \psi_{\mathrm{st}}\right)$ :

$$
\begin{aligned}
& \left(\frac{\gamma}{r_{\mathrm{st}}^{2}-1}+\frac{\epsilon_{1}}{2}\right)^{2}+\left(\frac{\omega_{0}}{1+r_{\mathrm{st}}^{2}}\right)^{2}=\frac{\epsilon_{2}^{2}}{4}, \\
& \tan \left(2 \psi_{\mathrm{st}}\right)=\frac{\omega_{0}\left(1-r_{\mathrm{st}}^{2}\right)}{\left(1+r_{\mathrm{st}}^{2}\right)\left(\gamma+\frac{\epsilon_{1}}{2}\left(r_{\mathrm{st}}^{2}-1\right)\right)} .
\end{aligned}
$$

The first equation implies that for a given $\epsilon_{1}$, no real $r_{\mathrm{st}}$ value, and hence, no SSS exist for $\epsilon_{2}=0$. In fact, for a given $\epsilon_{1}$, an SSS exists for $\epsilon_{2}$ larger than a critical value $\epsilon_{2 c}^{\mathrm{SSS}} \equiv \epsilon_{2 c}^{\mathrm{SSS}}\left(\epsilon_{1}\right)$. Alternatively, for a given $\epsilon_{2}$, there exists a critical $\epsilon_{1 c}^{\mathrm{SSS}}\left(\epsilon_{2}\right)$ beyond which the SSS exists.

\section{Standing Wave State (SWS):}

A standing wave state (SWS) is characterized by the order parameter $r(t)$ at long times oscillating as a function of $t$, but nevertheless yielding a non-zero time average at long times, $R \neq 0$. It is thus distinct from a synchronized stationary state (SSS) for which both the order parameter and its time average have a non-zero value at long times, but the former does not oscillate as a function of time. Deriving stability conditions for the SWS does not prove easy, unlike the ISS and the SSS. Hence, we analyzed using Eq. (23) the SWS stability by employing the numerical package XPPAUT [18. The period of the SWS is obtained by solving the time-dependent equations (23); we were however unable to obtain an analytical solution of the said equations.

In the next section, we view the above results vis-àvis results obtained from numerical integration of the dynamics (4) for large $N$.
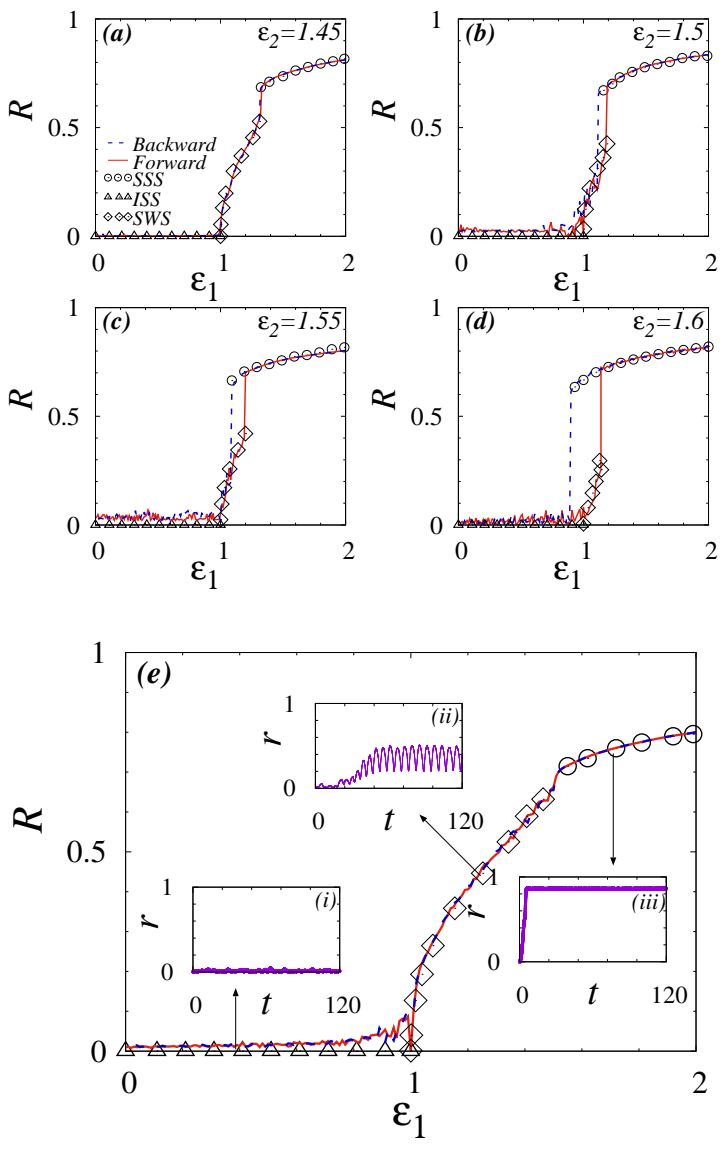

FIG. 3. The first four panels show the quantity $R$, namely, the time-averaged order parameter in the long-time limit, see Eq. 10, as a function of adiabatically-tuned $\epsilon_{1}$ and for four different values of $\epsilon_{2}$, see text. Panel (e) shows $r(t)$ as a function of $t$ for $\epsilon_{2}=1.4$ and for three representative $\epsilon_{1}$ values, namely, (i) $\epsilon_{1}=0.35$, when we have an ISS (see Fig. 2), (ii) $\epsilon_{1}=1.2$, when we have a SWS, and (iii) $\epsilon_{1}=1.7$, when we have a SSS. In all cases, the lines are obtained from numerical integration of the dynamics (4) for $N=5 \times 10^{5}$, while symbols correspond to predictions based on the OA-ansatzreduced dynamics 23) discussed in Section III The frequency distribution is a Lorentzian, see Eq. (7), with $\gamma=0.5$ and $\omega=1.0$.

\section{NUMERICAL RESULTS}

We start off this section with a discussion of results based on the OA-ansatz-reduced dynamics discussed in Section III and on direct numerical integration of the dynamics (4), in the case of the Lorentzian distribution (7) for the frequencies. The numerical integration involved use of a standard fourth-order Runge-Kutta integration algorithm with integration time step $\mathrm{d} t=0.01$. Within the OA-reduced dynamics, the analytical results we should be focusing on are Eq. (27) for the stability threshold $\epsilon_{1 c}^{\mathrm{ISS}}\left(\epsilon_{2}\right)$ of the ISS and Eqs. (29) and (30), with the latter yielding the existence threshold $\epsilon_{1 c}^{\mathrm{SSS}}\left(\epsilon_{2}\right)$ of the 


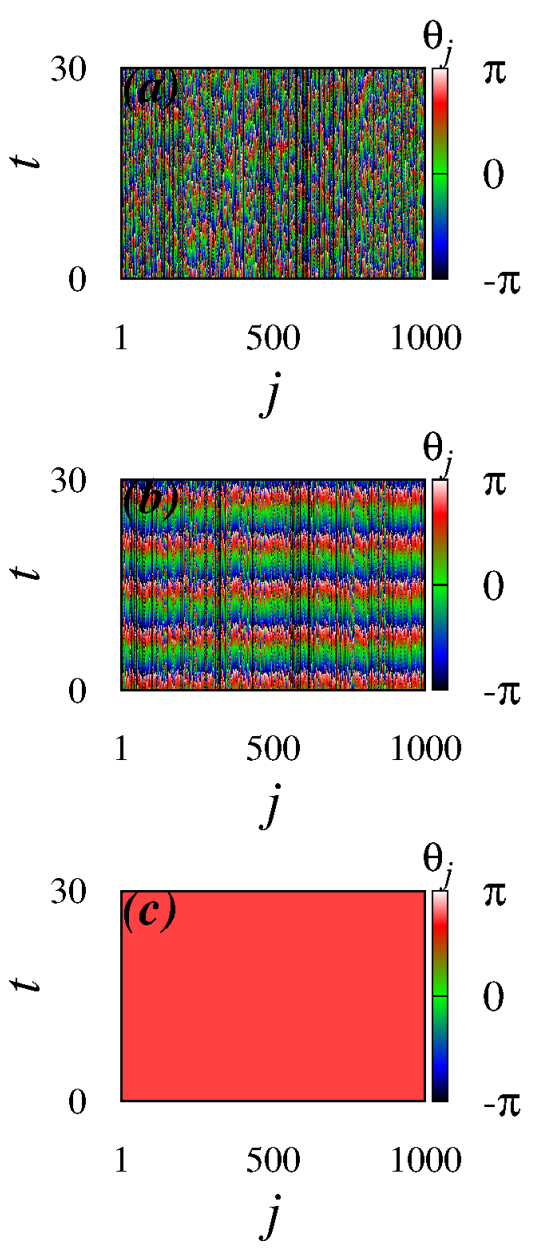

FIG. 4. Corresponding to the insets in panel (e) of Fig. 3 the figure shows $\theta_{j}$ for different $j$ and as a function of time at long times (the figure depicts the results not for the full range of $j$ but for $j=1,2, \ldots, 1000)$. The color coding given on the side of each panel denotes the intensity of $\theta$ values. The top panel corresponds to the ISS, the middle panel to the SWS and the bottom panel to the SSS. As may be seen from the figure, unlike the ISS and the SSS, the SWS exhibits a stationary wave pattern: at a fixed $j$, the value of $\theta$ changes periodically as a function of time. The data are obtained from numerical integration of the dynamics (4) with parameter values and other details same as in Fig. 3(e).

SSS, as we now detail. Choosing $\gamma=0.5$ and $\omega_{0}=1.0$ in Eq. (7), we may obtain $\epsilon_{1 c}^{\mathrm{SSS}}\left(\epsilon_{2}\right)$ for a fixed $\epsilon_{2}$ by varying $\epsilon_{1}$, from low to high values, using Eq. (29) to record the particular value of $\epsilon_{1}$ when for the first time the equation gives a solution for $r_{\mathrm{st}}$ in the range $0<r_{\mathrm{st}} \leq 1$, and identifying this particular value of $\epsilon_{1}$ with $\epsilon_{1 c}^{\mathrm{SSS}}\left(\epsilon_{2}\right)$. In Fig. 1] we show as a function of $\epsilon_{2}$ both the thresholds $\epsilon_{1 c}^{\mathrm{ISS}}\left(\epsilon_{2}\right)$ and $\epsilon_{1 c}^{\mathrm{SSS}}\left(\epsilon_{2}\right)$.

In Fig. 3, we show results based on numerical integration of the dynamics (4) with $N=5 \times 10^{5}$, for $R$ as a function of adiabatically-tuned $\epsilon_{1}$ for various values of $\epsilon_{2}$. We first let the system settle to the stationary state at a
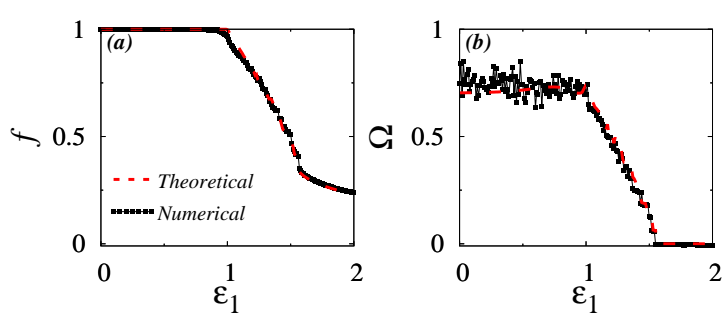

FIG. 5. Corresponding to the panel (e) in Fig. 3. the figure shows as a function of $\epsilon_{1}$ the mean-ensemble frequency $f$ and the mean-field frequency $\Omega$ at long times. Here, we have compared numerical integration results with those based on the OA ansatz; for details of computation, see Section IV]
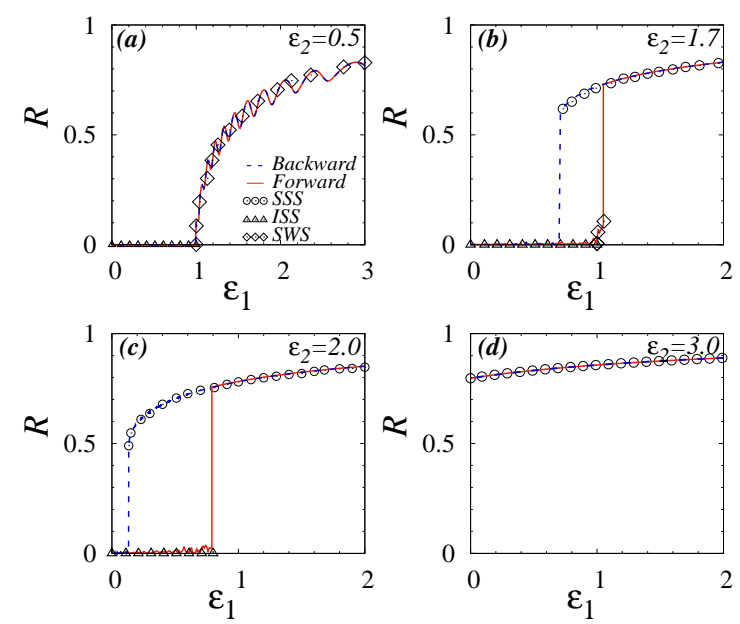

FIG. 6. The four panels show the quantity $R$, namely, the time-averaged order parameter in the long-time limit, see Eq. 10, as a function of adiabatically-tuned $\epsilon_{1}$ and for four different values of $\epsilon_{2}$, see text. In all cases, the lines are obtained from numerical integration of the dynamics (4) for $N=5 \times 10^{5}$, while symbols correspond to predictions based on the OA-ansatz-reduced dynamics 23) discussed in Section III The frequency distribution is a Lorentzian, see Eq. (7), with $\gamma=0.5$ and $\omega=1.0$,

fixed value of $\epsilon_{1} \approx 0$, and then tune $\epsilon_{1}$ adiabatically in time from low to high values while recording the value of $R$ in time; this corresponds to forward variation of $\epsilon_{1}$. Subsequently, we tune $\epsilon_{1}$ adiabatically in time from high to low values (backward variation of $\epsilon_{1}$ ). Adiabatic tuning ensures that the system remains in the stationary state at all times as the value of $\epsilon_{1}$ changes in time. In all panels in Fig. 3, we see that the system exists in either the ISS, the SWS or the SSS. For panel (a), the system exhibits a continuous transition between the ISS and the SWS, and between the SWS and the SSS. For panels (b), (c), and (d), the transition between the ISS and the SWS is still continuous, while the one between the SWS and the SSS becomes of first order. For $\epsilon_{2}=1.4$ case, we 


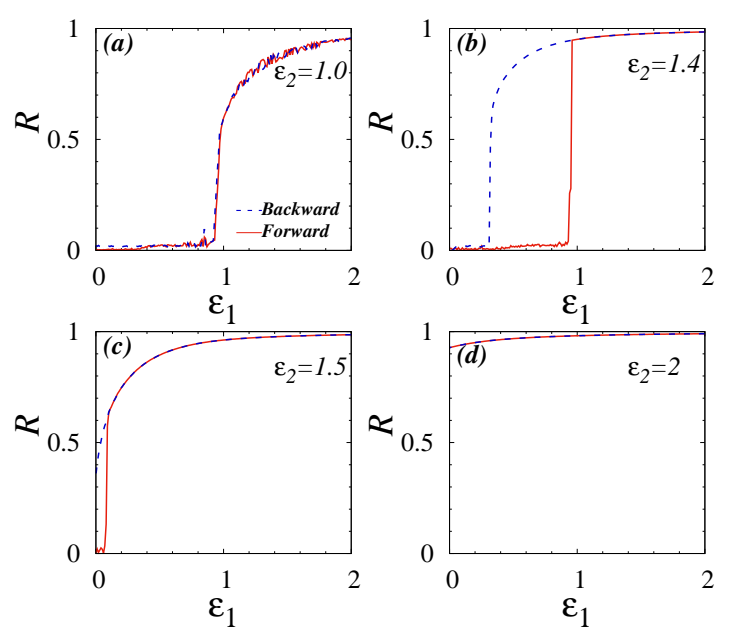

FIG. 7. The four panels show the quantity $R$, namely, the time-averaged order parameter $R(t)$ in the long-time limit, see Eq. 10, as a function of adiabatically-tuned $\epsilon_{1}$ and for four different values of $\epsilon_{2}$, see text. In all cases, the lines are obtained from numerical integration of the dynamics (4) for $N=5 \times 10^{5}$. The frequency distribution is a Gaussian, see Eq. (8), with $\omega_{0}=1.0$ and $\sigma=0.5$.

shows the variation of $R$ as a function of adiabaticallytuned $\epsilon_{1}$ via numerical integration of the dynamics (4) in Fig. 3(e); here, the three insets show for three representative values of $\epsilon_{1}$ the variation of $r(t)$ with time at long times. That the SWS is actually a standing wave is evident from the spatiotemporal plots in Fig. 4. where we show at long times the time series of $\theta_{j}$ for different $j$ in a system of size $N=10^{5}$ (the figure depicts the results not for the full range of $j$ but for $j=1,2, \ldots, 1000)$; the parameter values are the same as in Fig. 3(e). Here, the color coding refers to the intensity of $\theta$ values. It is evident from the figure that the SWS indeed behaves as a wave stationary in space, while the ISS and the SSS do not qualify as a wave.

Corresponding to Fig. 3(e), Fig. 5 shows as a function of $\epsilon_{1}$ the mean-ensemble frequency $f$ and the mean-field frequency $\Omega$ at long times. The numerical data plotted in the figure correspond to the following. First, one obtains $r_{x}$ and $r_{y}$ at long times from numerical integration of the dynamics (4) with parameter values and other details same as in Fig. 33(e). The values so obtained for $r_{x}$ and $r_{y}$ are then averaged over a stretch of time interval at long times; $f$ is then obtained as $f=\omega_{0}+2 \epsilon_{2} r_{x} r_{y}$. On the other hand, one obtains $\Omega$ numerically by substituting the numerically-obtained time-averaged values of $r$ and $\psi$ in Eq. 23). The figure also shows theoretical data for both $f$ and $\Omega$, which are obtained as follows: (i) for the ISS, we have $r_{x}=r_{y}=0$, giving $f=\omega_{0}$; (ii) for the SWS, we solve numerically Eq. 23) to obtain $r(t)$ and $\psi(t)$, from which we obtain $r_{x}(t)$ and $r_{y}(t)$ as $r_{x}(t)=r(t) \cos \psi(t), r_{y}(t)=r(t) \sin \psi(t)$. We then estimate $f$ from values of $r_{x}(t)$ and $r_{y}(t)$ averaged over time at long times; (iii) for the SSS, we obtain $r$ and $\psi$ at long times as being equal to the time-independent values $r_{\text {st }}$ and $\psi_{\text {st }}$, respectively, obtained by solving numerically Eq. (28). For $\Omega$, we have: (i) For the ISS, $\Omega$ is obtained by solving numerically for the time-dependent $\psi$ the second equation in 23) with $r=0$; (ii) For the SWS, $\Omega$ is obtained by solving numerically for $r$ and $\psi$ as a function of $t$ the coupled equations (23), and evaluating $\Omega$ as $\Omega=\mathrm{d} \psi / \mathrm{d} t$. (iii) For the SSS, we have $\Omega=0$. We see from the figure a very good match between numerical integration results and results based on the OA ansatz. For a discussion on the different behavior of mean-field and mean-ensemble frequencies in the context of the $\mathrm{Ku}$ ramoto model, we refer the reader to Ref. [21.

In Fig. 6, we show for four values of $\epsilon_{2}$ the variation of $R$ with $\epsilon_{1}$ adiabatically tuned over a wider range than is considered in Fig. 3 We see (i) in panel (a), for which $\epsilon_{1 c}^{\mathrm{ISS}}\left(\epsilon_{2}\right)$ is finite and $\epsilon_{1 c}^{\mathrm{SSS}}\left(\epsilon_{2}\right) \rightarrow \infty$ (see Fig. 1), no existence of the SSS and a continuous transition between the ISS and the SWS, (ii) in panel (b) the transition between the ISS and SWS is continuous, while the transition between the SWS and the SSS is first order, (iii) in panel (c) a first-order transition between the ISS and the SSS, and (iv) in panel (d), for which $\epsilon_{1 c}^{\mathrm{ISS}}\left(\epsilon_{2}\right)$ is finite and $\epsilon_{1 c}^{\mathrm{SSS}}\left(\epsilon_{2}\right) \rightarrow 0$, the existence of only the SSS. In all the panels in Figs. 3 and 6 , we see a good match of the data for $R$ obtained from numerical integration of the dynamics (4) and from the OA-ansatz-reduced-dynamics discussed in Section III. We have also checked the match between the OA-based results for $\epsilon_{1 c}^{\mathrm{ISS}}\left(\epsilon_{2}\right)$ and $\epsilon_{1 c}^{\mathrm{SSS}}\left(\epsilon_{2}\right)$ and those estimated from numerical integration of the dynamics (4). Our work thus provides further credence to the validity and the usefulness of the OA-ansatz in describing order parameter dynamics of globally-coupled phase oscillators, and is an useful addition to the evergrowing list of references demonstrating the applicability of the OA approach (Refs. 22, 25] provide a random sampling of papers on applications of the OA approach).

For the Gaussian frequency distribution, Eq. (8) with $\omega_{0}=1.0, \sigma=0.5$, Fig. 7 shows results for $R$ as a function of adiabatically-tuned $\epsilon_{1}$ for four values of $\epsilon_{2}$, obtained from numerical integration of the dynamics (4) with $N=$ $5 \times 10^{5}$. We see qualitatively similar phases as for the Lorentzian case, namely, the ISS, the SWS and the SSS, with (i) a continuous transition between the ISS and the SWS and between the SWS and the SSS in panel (a) a first-order transition between the ISS and th SSS in panels (b) and (c), and (iii) the existence of only the SSS in panel $(\mathrm{d})$.

\section{CONCLUSIONS}

In this work, we studied a nontrivial generalization of the celebrated Kuramoto model of spontaneous collective synchronization, by considering an additional interaction in the dynamics that breaks the rotational symmetry of the model. The Kuramoto model comprises limit- 
cycle oscillators of distributed natural frequencies that are coupled all-to-all. With the help of direct numerical integration of the dynamics and exact analytical results based on the so-called Ott-Antonsen ansatz for the specific case of a Lorentzian frequency distribution, we unraveled a rather rich phase diagram of the generalized model vis-à-vis the Kuramoto model. The phase diagram contains in it both stationary and standing wave phases. In the former, the synchronization order parameter $r$ has a long-time value that is time independent. On the other hand, one has in the standing wave phase an oscillatory behavior of the order parameter as a function of time that nevertheless yields a non-zero and time-independent time average. It would be interesting to study the effect of rotational-symmetry-breaking interaction on the inertial version of the Kuramoto model [14. Introducing inertia drastically modifies the phase diagram of the $\mathrm{Ku}-$ ramoto model, so we may already anticipate new features on adding the symmetry-breaking interaction. Investigations in this direction are under way and will be reported elsewhere.

\section{ACKNOWLEDGEMENTS}

The work of V.K.C. is supported by the SERBDST-MATRICS Grant No. MTR/2018/000676 and CSIR Project under Grant No. 03(1444)/18/EMRII. M.M. wishes to thank SASTRA Deemed University for research funds and extending infrastructure support to carry out this work. S.G. acknowledges support from the Science and Engineering Research Board (SERB), India under SERB-TARE scheme Grant No. TAR/2018/000023 and SERB-MATRICS scheme Grant No. MTR/2019/000560. He also thanks ICTP - The Abdus Salam International Centre for Theoretical Physics, Trieste, Italy for support under its Regular Associateship scheme.

\section{Appendix A: Motivating the form of the dynamics (4)}

Here, we motivate the form of the dynamics (4). To this end, let us consider a collection of $N$ globally- coupled Stuart-Landau limit-cycle oscillators with conjugate feedback, with dynamics given by

$$
\frac{\mathrm{d} z_{j}}{\mathrm{~d} t}=\left(1+\mathrm{i} \omega_{j}\right) z_{j}-\left|z_{j}\right|^{2} z_{j}+\frac{1}{N}\left[\epsilon_{1} \sum_{k=1}^{N}\left(z_{k}-z_{j}\right)-\epsilon_{2} \sum_{k=1}^{N} z_{k}^{*}\right]
$$

where the complex number $z_{j}$ characterizes the $j$-th oscillator, $j=1,2, \ldots, N$, the quantity $\epsilon_{1}$ denotes the strength of a diffusive coupling between the oscillators, while $\epsilon_{2}$ is the mean-field feedback strength. Writing $z_{j}$ in terms of real quantities $R_{j} ; 0 \leq R_{j} \leq 1$, and $\theta_{j} \in[-\pi, \pi]$, as $z_{j}=R_{j} e^{\mathrm{i} \theta_{j}}$, Eq. A1 gives

$$
\begin{aligned}
& \frac{\mathrm{d} R_{j}}{\mathrm{~d} t}=\left(1-\epsilon_{1}-R_{j}^{2}\right) R_{j} \\
& +\frac{1}{N}\left[\epsilon_{1} \sum_{k=1}^{N} R_{k} \cos \left(\theta_{k}-\theta_{j}\right)-\epsilon_{2} \sum_{k=1}^{N} R_{k} \cos \left(\theta_{k}+\theta_{j}\right)\right], \\
& \frac{\mathrm{d} \theta_{j}}{\mathrm{~d} t}=\omega_{j} \\
& +\frac{1}{N}\left[\epsilon_{1} \sum_{k=1}^{N} \frac{R_{k}}{R_{j}} \sin \left(\theta_{k}-\theta_{j}\right)+\epsilon_{2} \sum_{k=1}^{N} \frac{R_{k}}{R_{j}} \sin \left(\theta_{k}+\theta_{j}\right)\right] .
\end{aligned}
$$

In order to analyze the above dynamics, let us first consider the noninteracting case: $\epsilon_{1}=\epsilon_{2}=0$. It is then easily checked that Eq. A2 has fixed points $R_{j}=R_{\mathrm{s}} \equiv$ $1 \forall j$ and $R_{j}=R_{\mathrm{u}} \equiv 0 \forall j$, of which the former is stable and the latter is unstable. The long-time dynamics then corresponds to a limit-cycle for each of the individual oscillators with corresponding frequency $\omega_{j}$ and amplitude equal to $R_{\mathrm{s}}$, and with the corresponding motion described by the phase-only dynamics $\mathrm{d} \theta_{j} / \mathrm{d} t=\omega_{j} \forall j$. When the couplings $\epsilon_{1}$ and $\epsilon_{2}$ are sufficiently weak, a perturbation theory about the aforementioned limit-cycle behavior would imply substituting $R_{j}=R_{\mathrm{s}} \forall j$ in Eq. (A3), thus reducing it to a set of $N$ coupled equations of the form 4 .
[1] A. Pikovsky, M. Rosenblum and J. Kurths, Synchronization: a Universal Concept in Nonlinear Sciences (Cambridge University Press, Cambridge, 2001).

[2] J. Buck, Synchronous rhythmic flashing of fireflies. II., Q. Rev. Biol. 63, 265 (1988).

[3] C. S. Peskin, Mathematical aspects of heart physiology (Courant Institute of Mathematical Sciences, New York, 1975).

[4] I. Kiss, Y. Zhai and J. Hudson, Emerging coherence in a population of chemical oscillators, Science 296, 1676
(2002).

[5] A. A. Temirbayev, Z. Zh. Zhanabaev, S. B. Tarasov, V. I. Ponomarenko and M. Rosenblum, Experiments on oscillator ensembles with global nonlinear coupling, Phys. Rev. E 85, 015204(R) (2012).

[6] S. P. Benz and C. J. Burroughs, Coherent emission from twodimensional Josephson junction arrays, Appl. Phys. Lett. 58, 2162 (1991).

[7] Z. Néda, E. Ravasz, T. Vicsek, Y. Brechet and A. L. Barabási, Physics of the rhythmic applause, Phys. Rev. 
E 61, 6987 (2000).

[8] M. Rohden, A. Sorge, M. Timme and D. Witthaut, SelfOrganized synchronization in decentralized power grids, Phys. Rev. Lett. 109, 064101 (2012).

[9] R. Berner, J. Sawicki and E. Schöll, Birth and Stabilization of Phase Clusters by Multiplexing of Adaptive Networks, Phys. Rev. Lett. 124, 088301 (2020).

[10] Y. Kuramoto, Chemical Oscillations, Waves and Turbulence (Springer, Berlin, 1984).

[11] S. H. Strogatz, From Kuramoto to Crawford: exploring the onset of synchronization in populations of coupled oscillators, Physica D 143, 1 (2000).

[12] J. A. Acebron, L. L. Bonilla, C. J. P. Vicente, F. Ritort and R. Spigler, The Kuramoto model: a simple paradigm for synchronization phenomena, Rev. Mod. Phys. 77, 137 (2005).

[13] S. Gupta, A. Campa and S. Ruffo, Kuramoto model of synchronization: equilibrium and nonequilibrium aspects, J. Stat. Mech. R08001 (2014).

[14] S. Gupta, A. Campa and S. Ruffo, Statistical Physics of Synchronization (Springer, Berlin, 2018).

[15] E. A. Martens, E. Barreto, S. H. Strogatz, E. Ott, P. So and T. M. Antonsen, Exact results for the Kuramoto model with a bimodal frequency distribution, Phys. Rev. E 79, 026204 (2009).

[16] D. Iatsenko, S. Petkoski, P. V. E. McClintock and A. Stefanovska, Stationary and Traveling Wave States of the Kuramoto Model with an Arbitrary Distribution of Frequencies and Coupling Strengths, Phys. Rev. Lett. 110, 064101 (2013).
[17] D. Iatsenko, P.V.E. McClintock and A. Stefanovska, Glassy states and super-relaxation in populations of coupled phase oscillators, Nature Communications 5, 4118 (2014).

[18] B. Ermentrout, Simulating, Analyzing, and Animating Dynamical Systems: A Guide to XPPAUT for Researchers and Students (Society for Industrial \& Applied Math, Philadelphia, PA, 2002).

[19] E. Ott and T. M. Antonsen, Low dimensional behavior of large systems of globally coupled oscillators, Chaos 18, 037113 (2008).

[20] E. Ott and T. M. Antonsen, Long time evolution of phase oscillator systems, Chaos 19, 023117 (2009).

[21] S. Petkoski, D. Iatsenko, L. Basnarkov, and A. Stefanovska, Mean-field and mean-ensemble frequencies of a system of coupled oscillators, Phys. Rev. E 87, 032908 (2013).

[22] O. E. Omel'chenko, Partially coherent twisted states in arrays of coupled phase oscillators, M. Wolfrum and C. Laing, Chaos 24, 023102 (2014).

[23] C. R. Laing, Traveling waves in arrays of delay-coupled phase oscillators, Chaos 26, 094802 (2016).

[24] J. G. Restrepo and P. S. Skardal, Competitive suppression of synchronization and nonmonotonic transitions in oscillator communities with distributed time delay, Phys. Rev. Research 1, 033042 (2019).

[25] O E Omel'chenko, Traveling chimera states in systems of phase oscillators with asymmetric nonlocal coupling, Nonlinearity 33, 611 (2020). 